\title{
Erratum: A Case Study on Optimization of Biomass Flow During Single-Screw Extrusion Cooking Using Genetic Algorithm (GA) and Response Surface Method (RSM)
}

Tumuluru Jaya Shankar • Shahab Sokhansanj • Sukumar Bandyopadhyay $\cdot$ A. S. Bawa

Published online: 3 December 2010

(C) Springer Science+Business Media, LLC 2010

Erratum to: Food Bioprocess Technol (2010) 3:498-510

DOI 10.1007/s11947-008-0172-9

The original version of this article unfortunately missed the second affiliation of Shahab Sokhansanj. The missing affiliation is provided below.

The online version of the original article can be found at http://dx.doi. org/10.1007/s11947-008-0172-9.

T. J. Shankar $\cdot$ S. Sokhansanj

Department of Chemical and Biological Engineering,

University of British Columbia,

Vancouver, BC V6T1Z3, Canada

S. Sokhansanj

Environmental Sciences Division,

Oak Ridge National Laboratory,

Oak Ridge, Tennessee 37831, USA

S. Bandyopadhyay

Aquacultural Engineering Discipline,

Indian Institute of Technology,

Kharagpur, West Bengal, India

\section{A. S. Bawa}

Defence Food Research Laboratory,

Mysore, India

\section{T. J. Shankar $(\bowtie)$}

Department of Chemical and Biological Engineering,

University of British Columbia,

2360 East Mall,

Vancouver BC V6T 1Z3, Canada

e-mail: stumuluru@chml.ubc.ca

Present Address:

S. Bandyopadhyay

Faculty of Fishery Science, WBUFAS,

Kolkata, India 